\title{
Off-line handwritten Chinese character recognition based on GA optimization BP neural network
}

\author{
Huan Chen ${ }^{1}$, Xiangnian Huang ${ }^{2}$, Ping Cheng ${ }^{2}$, Yan $\mathrm{Xu}^{2}$ \\ ${ }^{1,2}$ Xihua University, Chengdu, China \\ Email:376369897@qq.com
}

\begin{abstract}
For the shortcomings of traditional BP neural network, which are easy to fall into local extreme and slow convergence, but the genetic algorithm has the characteristics of global optimization. We combine the two to form an optimized BP neural network algorithm. This method combines the advantages of neural networks and genetic algorithms, which can effectively improve the recognition rate and recognition speed of off-line handwritten Chinese character.
\end{abstract}

Keywords: Genetic algorithms; BP neural network; Handwritten Chinese character recognition

\section{Introduction}

Handwritten Chinese character recognition, especially the non-restrictive of freedom of writing handwritten Chinese character recognition is still considered to be one of the most difficult problems in the field of character recognition. To fundamentally improve the ability to identify the text, the most important are in two ways. The first is to extract characteristics which have stronger classification capabilities and high robustness; the second is to seek a more efficient, more accurate methods of classification and recognition $[1,2]$.
The genetic algorithm (Genetic Algorithm - GA) is a class of random search algorithm learning from biological natural selection and natural genetic mechanisms, its main feature is the group search strategy and the information exchange between the individuals in groups. Particularly, It is applied to solve the complex and non-linear problems, which is difficult to solve for traditional search method.

Bp neural network is multilayer feedforward networks which trained by error back propagation algorithm. BP neural network have hidden layer in the middle position, and have learning rules to follow, and these features make it has the ability to identify the non-linear model when training this network, so it is widely used in areas such as pattern recognition.

This paper effectively combine the advantages of the above two methods, and then propose a method of handwritten character recognition about BP neural network based on genetic algorithm optimization. First of all, make elastic mesh division of the image of Chinese characters after pre-treatment, and apply the grid to the refined image of Chinese characters, and then extract the eigenvectors of the four directions from the $\mathrm{H}, \mathrm{S}, \mathrm{P}, \mathrm{N}$; Then use genetic algorithm to get optimized weights and thresholds; Finally, train network using the optimized initial weights and thresholds, to predicted output [3]. 


\section{2 . Extraction of elastic mesh feature}

\subsection{Elastic mesh structure}

According to the strokes distribution of Chinese characters image, we use nonuniform network cable to divide the Chinese characters image, and get nonuniform grid, which is called elastic mesh. If $f(x, y)$ is the pixel value of Chinese characters image $(\mathrm{x}, \mathrm{y})$ with size $\mathrm{M} \times \mathrm{N}$, and the number of grid lines in horizontal and vertical direction respectively as N1, $\mathrm{N} 2$, then ,for any horizontal or vertical grid line $I_{i}, J_{j}, I_{k}, J_{k}$, it satisfies the following conditions:

$\int_{I_{i}}^{I_{i+1}} \int_{1}^{N} f(x, y) d x d y=\int_{I_{k}}^{I_{k+1}} \int_{1}^{N} f(x, y) d x d y=\frac{1}{N_{1}} \int_{1}^{M} \int_{1}^{N} f(x, y) d x d y$

$\int_{1}^{M} \int_{J_{i}}^{J_{i+1}} f(x, y) d x d y=\int_{1}^{M} \int_{J_{k}}^{J_{k+1}} f(x, y) d x d y=\frac{1}{N_{2}} \int_{1}^{M} \int_{1}^{N} f(x, y) d x d y$

Where, $f(x, y)$ represents the binary image of handwritten Chinese characters,

$$
f(x, y)= \begin{cases}1, & \text { black pixels } \\ 0, & \text { white pixels }\end{cases}
$$

The elastic grid of word "啊" is given in Figure 1.

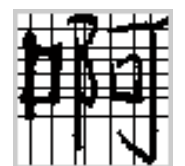

(a) $8 \times 8$

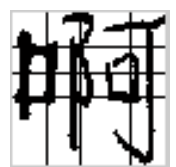

(b) $4 \times 4$

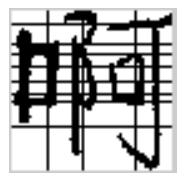

(c) $9 \times 4$
Fig. 1: Three kinds of elastic mesh of word “哬”

\subsection{The direction decomposition and the direction feature extraction of Chinese characters skeleton}

In This article we select the method of skeleton direction decomposition, which based on the extraction of the skeleton of Chinese characters, and decompose the Chinese character for four directions in $\mathrm{H}$, $\mathrm{S}, \mathrm{P}, \mathrm{N}$. Specific guidelines are as follows:

\begin{tabular}{|c|c|c|}
\hline P4 & P3 & P2 \\
\hline P5 & P & P1 \\
\hline P6 & P7 & P8 \\
\hline
\end{tabular}

Fig.2: 8 neighborhood of $P$

- If P1 and P5 are black pixels, then p belongs to the component of $\mathrm{H}$.

- If P3 and P7 are black pixels, then p belongs to the component of $\mathrm{S}$.

- If P2 and P6 are black pixels, then p belongs to the component of $\mathrm{P}$.

- If P4 and P8 are black pixels, then $\mathrm{p}$ belongs to the component of $\mathrm{N}$.

Assume that we get grid $\Omega_{1}, \Omega_{2}, \ldots, \Omega_{n}$, and after decompose Chinese characters into four directions in $\mathrm{H}, \mathrm{S}, \mathrm{P}, \mathrm{N}$., we take $f_{H}(x, y) 、 f_{V}(x, y) 、 f_{L}(x, y) 、 f_{R}(x, y)$ to represent sub-image of decomposition Chinese characters in four directions Respectively., then probability distribution characteristics of elastic grid pixel in each direction can be given by the following formulas:

"H" direction:

$$
m_{H}^{i}=\frac{\iint_{\Omega_{i}} f_{H}(x, y) d x d y}{\iint f(x, y) d x d y}
$$

"S" direction: 


$$
m_{S}^{i}=\frac{\iint_{\Omega_{i}} f_{S}(x, y) d x d y}{\iint f(x, y) d x d y}
$$

"P" direction:

$$
m_{P}^{i}=\frac{\iint_{\Omega_{i}} f_{P}(x, y) d x d y}{\iint f(x, y) d x d y}
$$

"N" direction:

$$
m_{N}^{i}=\frac{\iint_{\Omega_{i}} f_{N}(x, y) d x d y}{\iint f(x, y) d x d y}
$$

Where, $i=1,2, \cdots n, \mathrm{n}$ is the number of the grid. If we combined characteristics of four directions we can get an integral feature vectors of a Chinese character.

\section{Genetic algorithm to optimize BP neural network}

The genetic algorithm to optimize BP neural network is divided into three parts. They are BP neural network structure determination, genetic algorithm optimization and BP neural network prediction output.

\subsection{Genetic algorithm to optimize weights and threshold of neural network}

Genetic algorithm steps are as follows:

- Initialize population randomly, crossover probability is $p_{c}$, mutation probability is $p_{m}$, iteration for 100 times.

- Calculate the fitness value of each individual, to find the best individual, fitness function is as follows:

$$
f(i)=1 / E(i)
$$

$$
E(i)=\sum_{j=1}^{N}\left(V_{i j}-T_{j}\right)^{2}
$$

Where, $\mathrm{N}$ is the number of samples, $V_{i j}$ is the output of the i-th chromosome encoding, $T_{j}$ for the desired output.

- Select operation, using roulette method to select fitness good individuals from the population to form a new population, the selection probability $p_{i}$ of each individual $\mathrm{i}$ is

$$
P_{i}=\frac{f_{i}}{\sum_{j=1}^{p} f_{j}}
$$

Crossover operation, using real numbers crossing method, then the operating method of k-th chromosome and i-th chromosomes in the intersection of the $\mathrm{j}$ bit is as follows:

$$
\begin{aligned}
& G_{k j}=G_{k j}(1-b)+G_{l j} b \\
& G_{l j}=G_{l j}(1-b)+G_{k j} b
\end{aligned}
$$

Where, $b$ is a random number in $[0,1]$.

- Mutation operation, Mutation operating the $\mathrm{j}$-th gene of the selected $\mathrm{i}$-th individual are as follows:

$$
G_{i j}=\left\{\begin{array}{l}
G_{i j}+\left(G_{i j}-G_{\max }\right) * f(g), r \geq 0.5 \\
G_{i j}+\left(G_{\min }-G_{i j}\right) * f(g), r<0.5
\end{array}\right.
$$

Where, $G_{\max }$ is the upper bound of the gene $G_{i j}, G_{\min }$ for the lower bound of the gene $G_{i j}, f(g)=r^{\prime}\left(1-g / D_{\max }\right)$, and $\mathrm{g}$ for the current number of iterations.

- Insert the produced new individual into the population, and to calculate the fitness of the new individual. 
- Determine whether evolution ended, and if not, return to step 3 .

When the satisfied accuracy is reached, decoding the obtained optimum individual, then the weights and threshold value of the network after optimization can be obtained[4].

\subsection{Prediction output of BP neural network}

In Matlab environment, the neural network toolbox provides a dedicated function for establishing neural network. Training network after created BP network. For trained BP network, you can use the simulation function to predict the forecast data.

\section{Results}

In order to test the effectiveness of the proposed algorithm, we selected a set of 100 samples of 160 Chinese characters in GB2312-80 level font in hcl2000 handwritten character sample library. Of these, 70 sets are taken as training samples, 30 sets as test samples. In the experiment, we take traditional Bp neural network and Bp neural network optimized by genetic algorithm to do output identification for Chinese character sample respectively. The recognition results are as follows:

Table I: Recognition Results of Different Neural Network

\begin{tabular}{|c|c|c|}
\hline $\begin{array}{c}\text { Recognition } \\
\text { method }\end{array}$ & $\begin{array}{l}\text { Iteration } \\
\text { times }\end{array}$ & $\begin{array}{l}\text { Recognition } \\
\text { rate }\end{array}$ \\
\hline $\begin{array}{c}\text { Traditional } \\
\text { BP neural } \\
\text { network }\end{array}$ & 1200 & $89.6 \%$ \\
\hline $\begin{array}{c}\text { Optimized } \\
\text { BP neural } \\
\text { network }\end{array}$ & 143 & $85.4 \%$ \\
\hline
\end{tabular}

\section{Conclusion}

The experimental results show that the optimized BP neural network algorithm decrease the number of iterations than traditional BP neural network on learning and training, and the recognition rate has corresponding increase. In short, the design of the classifier is the difficulties of off-line handwritten Chinese character recognition, and the combination of genetic algorithms and BP neural network can effectively improve the rate of recognition of handwritten Chinese characters.

\section{References}

[1] X.N. Huang. "An multiple classifiers integrated system of On-line natural handwritten Chinese characters recognition".Journal of Chinese Information Processing.2000,vol14(5),pp37-41.

[2] Y.F. Sun, Y. Chen, Y.Z. Zhang. "symmetry-based Recognition method for similar Chinese characters". Journal of Chinese InformationProcessing. 2004,vol18(2),pp51-57.

[3] B Jia., X.D. Tian,F.Yang. "Off-line handwritten Chinese character recognition based on double contour feature". 2009International symposium on intelligent information systems and applications,China,pp399-402, October,.2009.

[4] T.H. Su, T.W. Zhang, D.J. Guan. "Off-line recognition of realistic Chinese handwriting using segmentation-free strategy". Pattern Recognition, 2009,vol42,pp167-182. 